\title{
Los límites del espionaje estatal: archivos de inteligencia y organización obrera en la expulsión del grupo Albatros del Astillero Río Santiago (1992)
}

\section{The limits of state espionage: files of intelligence and worker organization in the expulsión of the Albatros group (Río Santiago Shipyard, 1992)}

\author{
Iván Knopoff*
}

«Todas las policías (...) son casi del todo impotentes»

Resumen: Los conflictos obreros fueron registrados por los servicios de inteligencia del Estado, es el caso de la Dirección de Inteligencia de la Policía de la Provincia de Buenos Aires (DIPPBA). Produjeron análisis sobre posibles escenarios. Para esto, infiltraron distintos niveles de las organizaciones de masas. Sin embargo, cuando no lograban adentrarse en las esferas de dirección sindical o política, los análisis no se ratifican en la realidad. Es el caso del Astillero Río Santiago de Ensenada cuando el grupo naval Albatros ocupa la planta y es repelido por los trabajadores.

Palabras Clave: movimiento obrero, conflicto obrero, privatizaciones, servicios de inteligencia

\begin{abstract}
Worker's struggle were recorded by the State's intelligence services, as in the case of the DIPPBA of the province of Buenos Aires. They produced analyzes on possible scenarios. For this, they infiltrated different levels of mass organizations. However, when they were unable to penetrate into the spheres of union or political leadership, the analyzes are not ratified in reality. This is the case of the Río Santiago de Ensenada Shipyard when the naval group Albatros occupies the plant and is repelled by the workers.
\end{abstract}

Keywords: labor movement, worker's struggle, privatizations, intelligence services

Recibido: 17 febrero 2018 Aceptado: 28 mayo 2018

\footnotetext{
* Argentino. Profesor de Historia y maestrando de la Maestría en Historia y Memoria de la Facultad de Humanidades y Ciencias de la Educación de la Universidad Nacional de La Plata. Correo electrónico: Ivan.knopoff@gmai.com
} 
El presente artículo parte de preguntarse acerca de la relación entre los informes de los servicios de inteligencia y las acciones del gobierno, en este caso nacional. La investigación se encuadra en un proyecto de tesis de maestría sobre las formas de organización y de lucha de los trabajadores del Astillero Río Santiago ${ }^{1}$ (ARS) frente a los intentos de privatización.

En septiembre de 1992 el gobierno nacional definió el cierre de la empresa a través de la ocupación militar de la misma y la apertura de masivos retiros voluntarios. Los servicios de inteligencia de la ex Dirección de Inteligencia de la Policía de la Provincia de Buenos Aires (DIPPBA) registraron el conflicto detalladamente; el reservorio documental se encuentra en propiedad de la Comisión Provincial por la Memoria. Los documentos del caso están compilados en una serie específica: Fondo DIPPBA División Central de Documentación, Registro y Archivo, Mesa B, Factor Gremial, Carpeta 39, Legajo 43, Tomo IV.

Los archivos de la DIPPBA deben entenderse como "archivos de represión", en el sentido que fueron elaborados por parte del aparato de seguridad del Estado como forma de seguimiento y control social ${ }^{2}$; cuentan con cerca de cuatro millones de folios sobre conflictos sindicales, asociaciones civiles y culturales y organizaciones de la esfera de la sociedad civil ${ }^{3}$.

Para el interés de este artículo en particular, retomamos los planteos de Pablo Ghigliani ${ }^{4}$ acerca de la utilidad de estos materiales en la realización de una historia social del trabajo. La reconstrucción que hace el autor permite observar la importancia que la inteligencia del Estado daba a las instancias de organización colectiva: «cerca de un 40\% de la documentación versa sobre instancias colectivas de discusión, propaganda y debate sobre problemas y políticas gremiales. En ellos aparece información sobre conflictos, salarios, condiciones de trabajo, disputas intra-sindicales, políticas patronales, racionalizaciones productivas, despidos, suspensiones, negociaciones, etc.» ${ }^{5}$.

En este trabajo se busca intentar entender si el gobierno no preveía la posibilidad de confrontación física entre las fuerzas de seguridad y los trabajadores cuando insta al Ministerio de Defensa, como se verá más adelante en el texto, a ocupar militarmente las instalaciones del Astillero Río Santiago. El Albatros -grupo dependiente de Prefectura Naval Nacional, seleccionado para esa misión - no es un grupo entrenado para conflictos sindicales, sino para combatir el narcotráfico y el terrorismo ${ }^{6}$. Un año antes la privatización de la siderúrgica $\mathrm{SOMISA}^{7}$ debió pasar por la ocupación de planta por parte de la gendarmería. En aquella ocasión, los trabajadores también acamparon en las inmediaciones, pero no se enfrentaron a la fuerza de ocupación. ¿Entendía el gobierno que volvería a repetirse un accionar similar por parte de los trabajadores? SOMISA formaba parte de las industrias estratégicas en el proceso

${ }^{1}$ Ubicado en la ciudad de Ensenada, provincia de Buenos Aires, es la empresa naval más importante del país.

2 Al respecto véase María Eugenia Marengo, "Los mecanismo del control social: el caso de la ex DIPBA". Derecho y Ciencias Sociales $\mathrm{N}^{\circ} 4,2011$

3 Pueden revisarse los trabajos de Emmanuel Kahan, “¿Qué represión, qué memoria? El "archivo de la represión” de la DIPBA: problemas y perspectivas". Question N¹6, 2006; Magdalena Lantieri y Mariana Nazar, "A 10 años de la apertura del Archivo de la DIPPBA". Aletheia N9, 2014; Ana Cecilia Solari Paz "Estado, politica y sociedad en una Argentina en crisis (1955-1989)", 2016.

4 Pablo Ghigliani, “Archivos policiales e historia social del trabajo (1957-1977)”. VII Jornadas de Sociología UNLP. 2012

5 Ghigliani op. cit. Pág 10

${ }^{6} \mathrm{Al}$ respecto puede verse el sitio oficial en: http://www.prefecturanaval.gov.ar/web/es/html/inst servicios9.php

${ }^{7}$ Sociedad Mixta Siderúrgica Argentina. Actualmente parte del conglomerado Techint 
de privatizaciones, junto a YPF, los ferrocarriles y los servicios públicos. El proceso de liquidación de activos nacionales ${ }^{8}$ tuvo su epicentro en estas principales empresas del país. El Astillero Río Santiago -la industria naval en general- se encontraba en retroceso desde los años '80 y no era un factor de alta rentabilidad para el gran capital como sí otras empresas estatales. Esto podría explicar por qué el cierre sucede 3 años después de la sanción de la ley 23696 de Reforma del Estado y dos años posteriores al decreto 1398/90 que incorporaba las empresas del área de Defensa, entre ellas Astilleros y Fabricaciones Navales del Estado (AFNE) del cual dependía el ARS, a dicha ley.

En un primer apartado se verá el escenario general de las reformas estructurales; en segundo lugar, el propio planteo del gobierno de cerrar el ARS; los apartados tercero y cuarto se referirán, respectivamente, al análisis realizado por los agentes de la DIPPBA sobre el conflicto y la orientación de enfrentar el cierre del Astillero por parte de los trabajadores.

Se intentará adentrar en los documentos elaborados por los agentes de la DIPPBA con el objeto de echar luz sobre esta problemática del accionar de la patronal ante el proceso de cierre del Astillero. Si bien se realiza un acercamiento a algunas consideraciones que los trabajadores extraen de esta lucha contra la privatización, como forma de contraste con las apreciaciones de los agentes de inteligencia, resta para otras investigaciones ahondar en las perspectivas de aquellos trabajadores que fueron los protagonistas de los sucesos.

\section{El gobierno}

El gobierno nacional, administrado por Carlos Menem desde julio de 1989, desenvolvió el programa de las reformas estructurales bajo lo que se conoce como Consenso de Washington: desregulación financiera; liberalización del comercio y su regulación a partir del mercado; privatización de las empresas del Estado; una reforma impositiva que reduce presiones tributarias a los grandes conglomerados y amplia las bases de la recaudación; y la reducción del llamado "gasto público" 9 . Este programa de reformas no fue monopolio exclusivo del partido de gobierno, el Partido Justicialista (PJ), sino también por la principal oposición, la Unión Cívica Radical (UCR), y también fuerzas menores. El principal armazón de las reformas fueron las leyes 23696 de Reforma del Estado y 23697 de Emergencia Económica; la primera habilitó al Poder Ejecutivo Nacional (PEN) a que el obrase por decreto en la privatización y/o disolución de empresas y la segunda habilitó a proceder mediante decretos en todas las reformas señaladas. Finalmente, la Ley de Convertibilidad en 1991 estableció la paridad cambiaria 1 peso 1 dólar por ley.

Este apoyo de los partidos mayoritarios se debió al hecho de que compartían ese programa como única alternativa ante la crisis económica y social -la hiperinflación-con que sucumbió el gobierno radical de Alfonsín. Como señala Alberto Bonnet, la hiperinflación como fenómeno produjo una coerción de tipo económica sobre amplias capas de la población. Si

$8 \mathrm{Al}$ respecto de la liquidación de los activos nacionales puede verse Alberto Bonnet, "La hegemonía menemista", 2008; Daniel Azpiazu "Las privatizadas ayer y hoy", 2005.

9 Véase Oscar Oszlak "El mito del Estado mínimo". Desarrollo Económico vol $42 N^{\circ}$ 168, 2003; Atilio Borón y Mabel Thwaites Rey "La expropiación neoliberal. El experimento privatista en la Argentina". En James Petras y Henry Veltmeyer, Las privatizaciones y la desnacionalización de América Latina, 2004; Alberto Bonnet, "La hegemonía menemista", 2008. 
para los capitalistas implicó la posibilidad de obtención de ganancias extraordinarias, para los trabajadores puso en cuestión la propia capacidad de supervivencia ${ }^{10}$. Menem fue reelecto en 1994 y el programa de la Convertibilidad continuó como tal en 1999, esta vez bajo el gobierno de la Alianza. En un período acotado de tiempo, entre 1990 y 1991, las principales empresas del país fueron privatizadas: YPF, FFCC, SOMISA, Entel, Segba, etc. El proceso implicó una fuerte reducción de trabajadores ocupados, pero también un ingreso de divisas que permitieron en ese mismo período alcanzar la inflación $0 \%$, base de sustentación de la Convertibilidad. La inflación controlada operó como el sustrato económico necesario para evitar el desenvolvimiento de enfrentamientos del conjunto de la clase trabajadora a las privatizaciones.

Otro de los fenómenos de las reformas estructurales fue la descentralización del Estado, que significó cambios de jurisdicción de áreas, como la educación o la salud, de la órbita nacional a las provinciales, así como la creación de entes reguladores de las empresas a ser privatizadas. Privatizaciones y descentralización, permiten pensar aquello que explica Adrián Piva ${ }^{11}$ acerca de que las luchas obreras que acontecieron en el período fueron vastas, pero en escalas locales y regionales ${ }^{12}$, en un claro desacople de las grandes manifestaciones obreras -huelgas generales, movilizaciones- que a principios de los años '90 son casi nulas. El intento de privatización/cierre del Astillero Río Santiago debe encuadrarse en este escenario general.

$$
\text { El "cierre" }
$$

Descripta la orientación política general del oficialismo, se analizará el caso del Astillero.

La producción naval tiene un fuerte componente artesanal y requiere de una mano de obra altamente calificada ${ }^{13}$. No opera de forma seriada, sino bajo contratos. Es un trabajo a pedido y no puede reestructurarse hacia una gran escala. Desde el gobierno de Alfonsín, la aprobación de créditos para realizar los contratos que la empresa ganaba fue dilatándose. Sin los créditos, la posibilidad de iniciar la construcción naviera es prácticamente imposible. El rol del Estado en esta industria es esencial dado su papel como regulador, promotor, productor, demandante, proveedor de infraestructura y articulador de mecanismos de financiamiento ${ }^{14}$. En el contexto de las reformas estructurales, operaba en el sentido opuesto mediante la desregulación del sector naval, dejando sin efecto medidas de promoción de la actividad; la liberación del

\footnotetext{
10 Bonnet, op. Cit. Página 193.

11 Adián Piva, "El desacople entre los ciclos del conflicto obrero y la acción de las cúpulas sindicales en Argentina (1989-2001)". Estudios del Trabajo N³1, 23-52, 2006.

${ }_{12} \mathrm{Al}$ respecto de algunas luchas obreras emblemáticas del período, pueden verse los estudios de casos para YPF de Hernan Palermo, "Cadenas de oro negro en el esplendor y ocaso de YPF". Buenos Aires: Grupo Antropología del Trabajo, 2012; Fernanda Torres, "La privatización de YPF en Comodoro Rivadavia. Algunas características y consecuencias sociales y laborales". Trabajo y Sociedad, 279-295, 2011; Pablo Jaitte, "La lucha de los petroleros contra la privatización de YPF: Un intento de sintesis". La Plata: VI Jornadas de sociología UNLP, 2010; para el caso de Hipasam: Fernando Aiziczon, "Trayectorias militantes, izquierda y política sindical: la intervención del MAS en Sierra Grande a través de las vivencias de un obrero minero, Patagonia Argentina (1988-1991)". Izquierdas $N^{\circ} 31$, 46-70, 2016. (Aiziczon, 2016); entre tantos.

${ }^{13}$ Cintia Russo "Astillero navales del Estado". XXII Jornadas de Historia Económica - Río Cuarto, 2010, página 11

14 Ver Lucía Mercedes Mauro, "La industria naval como un sector estratégico: análisis del rol Estado". VI Jornadas de Jóvenes Investigadores, 2011
} 
mercado de embarcaciones, permitiendo la importación de buques libre de gravámenes; y privatizando empresas armadoras y astilleros públicos ${ }^{15}$.

Sobre esto los trabajadores realizaron denuncias tanto bajo el alfonsinismo como durante el menemismo. En 1987 los trabajadores, a través del secretario general de la Asociación de Trabajadores del Estado (ATE) seccional Ensenada, Ismael Barros, denunciaron que la traba a esos créditos implicó el fracaso de un contrato para fabricar buques marroquíes ${ }^{16}$; en julio de 1992, dos meses antes de la ocupación del Albatros y el "cierre" de la fábrica, el cuerpo de delegados, ATE-Ensenada, Provincia de Buenos Aires y ATE Nación, publicaron una solicitada que salió en el Diario El Día donde se responsabilizaba al gobierno por la falta de trabajo debido a que: «un contrato que se tenía preadjudicado, para construir 6 buques para Holanda, se perdió al no autorizar el Gobierno Argentino un aval de u\$d 4800000 para comenzar su construcción, lo que motivó que 3 de esos buques se estén construyendo en el astillero brasileño Verolme» (El Día, 10/7/1992).

Al bloqueo de créditos para realizar los contratos internacionales se sumó la falta de producción nacional tanto para YPF como para la Marina Mercante. Un armazón específico de leyes navales fue reduciendo la importancia de la flota de bandera nacional en el comercio exterior. Para 1991, el Astillero se encontraba casi paralizado. Entre 1989 y 1993 el 60\% ${ }^{17}$ de los trabajadores del ARS aceptó el retiro voluntario; a pesar de esto, el gobierno no logró una aceptación absoluta de la necesidad de aplicarse las reformas productivas ${ }^{18}$.

El 19 de septiembre de 1992 se difundió un comunicado de Astilleros y Fábricas Navales del Estado (AFNE) informando el cierre del Astillero Río Santiago (ARS) «ante la imperiosa necesidad de encarar una reestructuración de la empresa» ${ }^{19}$. La copia del comunicado que los agentes de la DIPPBA fotocopian no posee fecha precisa más allá de septiembre de 1992; esa situación tal vez pueda indicar que se encontraba escrito desde tiempo antes, a la espera del momento preciso para su difusión.

Dicho comunicado explicitaba que esta "reestructuración" implicaba el receso operativo por 45 días -desde el lunes 21 de septiembre-; se dispensaba de funciones a todo el personal a excepción de guardias y quienes liquidan sueldos; el pago de los sueldos íntegros y, entre el 28 de septiembre y el 3 de octubre, la apertura del libro de retiros voluntarios. Lo único que seguiría en funcionamiento normales era la Escuela Técnica del ARS (ETARS).

Para garantizar el cierre del Astillero, el PEN dio la orden al Ministerio de Defensa de impedir el ingreso de los trabajadores a la fábrica. Así, el grupo de élite Albatros, junto al Batallón de Infantería de Marina $\mathrm{N}^{\circ} 3$, ocupó la fábrica con un número que osciló entre los 50 y los 200 efectivos dependiendo si relatan los trabajadores, la DIPPBA o el diario El Día. Los Albatros ingresaron por el río al cierre de la jornada, cuando habían salido todos los

\footnotetext{
${ }^{15}$ Juliana Frassa, "Políticas públicas de desregulación y sus consecuencias sobre la producción y el empleo. El caso de la política naviera y la industria naval argentina. Informe IEFE, 39-51, 2006

${ }^{16}$ El Día 19/10/1987

${ }_{17}$ De 2548 trabajadores en 1989 se produce una reducción hasta llegar a 1036 en 1993 (Frassa, 2009, pág. 108)

${ }^{18}$ Esta situación si ocurrió en otras fábricas, como por ejemplo Propulsora Siderúrgica; al respecto véase María Alejandra Esponda "La reestructuracion productiva de los 90 en Propulsora Siderúrgica: debates, formas de organización y disputas de poder". En V. Basualdo, La clase trabajadora argentina en el siglo XX: experiencias de lucha y organización (págs. 325-361), 2011.

${ }_{19}$ Mesa B, Factor Gremial, Carpeta 39, Legajo 43, Tomo IV
} 
trabajadores y solo quedaba el reducido turno noche. Una parte considerable de trabajadores los miembros de la Mesa de Delegados, la conducción del sindicato- se encontraban en el Pasaje Dardo Rocha en el acto de la Industria Naval que se había pasado una semana por problemas técnicos en la edición final de un corto que fue proyectado ese mismo día titulado Luchar vale la pena. El acto se convirtió en una asamblea donde confluyeron, junto a los obreros del Astillero, otros sindicatos, organizaciones estudiantiles y partidos políticos. El escenario, que iba a ser utilizado para presentar distintas partes del acto, sirvió de tribuna para convocar a la asamblea del día siguiente en la plaza Belgrano -plaza central de Ensenada. Así, a las 10hs del sábado 19 de septiembre, en asamblea, los trabajadores resolvieron una serie de puntos:

«1) que se retiren inmediatamente del Astillero la totalidad de las fuerzas de seguridad con el objeto de que puedan ingresar libremente los trabajadores a la planta; 2) que se proceda a efectuar una asamblea de ATE Provincia y ATE Nación con el fin de procurar un inmediato paro regional y nacional; 3) convocar a los delegados de Petroquímica (Mosconi), Propulsora (Siderúrgica), trabajadores del Estado y políticos con el fin de lograr la adhesión ante el cierre del ARS; 4) convocar a todos los trabajadores con el fin de que junto a sus familias se presenten el día lunes a las 06:30hs en el ARS con el objeto de preservar y defender las fuentes de trabajo; 5) que los delegados de planta y ATE Ensenada, a partir de la fecha, se instalaran en carpas frente al lugar hasta tanto se encuentren soluciones al problema, convocando a aquellos trabajadores que quieran permanecer en el sitio, para el día de mañana (domingo 20) a las $15 \mathrm{hs} ; 6$ ) proceder a la inmediata creación de un fondo de huelga» (Mesa B, Factor Gremial, Carpeta 39, Legajo 43, Tomo IV)

A las 4:30hs de la madrugada del lunes 21 de septiembre, tras una reunión entre el gobernador y el intendente de Ensenada Adalberto Del Negro, las fuerzas que ocupaban el Astillero salieron y los trabajadores ingresaron en su horario normal de trabajo ¿En aquella reunión entre el intendente de la ciudad y el gobernador se discutió la posibilidad concreta de que los trabajadores ingresasen y se produjese un enfrentamiento entre ellos y las fuerzas de ocupación? Si bien la respuesta a esta pregunta es compleja de analizar, la observación sobre el papel de los agentes de inteligencia de la provincia arroja algunas luces

\section{El espionaje}

Los agentes de la DIPPBA elaboraron más de 2000 folios desde la fundación del Astillero, en 1953, hasta la apertura de sus archivos en 1998. La Carpeta 39 Legajo 43, Tomo IV se titula “Astillero Río Santiago: 02-01-1992 al 25-09-1992” y está compuesta por 315 folios, de los cuales 48 refieren directamente al momento de ocupación de la fábrica por parte del grupo Albatros. Fueron diversos agentes los que produjeron información sobre este hecho particular. Analíticamente se ordenarán como tres tipos de informes diferentes, cada uno propio de uno ¿O varios? - informante. El primero catalogado en Factor Gremial, el segundo en Factor Laboral, y el tercero también como Factor Laboral pero seguido en la subsección otras medidas de acción directa. 
Pablo Ghigliani ${ }^{20}$ alerta sobre la posibilidad de que varios de los informes, en términos generales, no sean producto de un único agente, sino de un equipo. Resolver esta inquietud no es válido con el actual conocimiento que se tiene sobre el funcionamiento de ese aparato de inteligencia estatal; debido eso, serán considerados como un agente único para cada secuencia. Para el fin de este artículo, se utilizará el Factor de identificación de cada informe como una unidad dadas las particularidades de los mismos -cada uno inicia retomando información suministrada en uno previamente elevado. El investigador Felipe Venero señala que, sobre la construcción de los informes:

«Los legajos sobre los conflictos se confeccionaban a partir de la información que llegaba a la central sobre el hecho. (...) Una vez que se despertaba el interés se mantenía un seguimiento constante de la situación, que incluso se traducía en varios partes diarios en los que se enviaba, desde las regionales, un seguimiento minucioso de lo que iba sucediendo durante el día. Los expedientes se cerraban una vez terminado el conflicto, y no mantenían un rastreo posterior ante la posibilidad de que la situación se reanudase» ${ }^{21}$

El agente que construyó el informe de Gremial fue el único que elaboró uno del día previo a la ocupación de la fábrica. En cuanto al día 18, el mismo agente realizó dos partes, uno de la mañana y otro posterior a las 21:30hs. Existe un informe del 19 y un informe del día 21 de septiembre que da cuenta de lo sucedido también el día anterior, domingo 20. En cuanto a Laboral, se compone por dos informes del 18, uno del 19 y uno del 21 que también da cuenta del día anterior. Finalmente, de Acción Directa solo disponemos del 19 dos informes y uno del 21. En términos de la semana, 18 de septiembre fue viernes, 19 sábado, 20 domingo y 21 lunes; que no existan informes con fecha $20 / 9$ indica que ese domingo ninguno de estos agentes operó; y es posible que fuese un día libre para ellos.

El agente que elaboró el primer informe Gremial con fecha del 18 de septiembre consideraba que los trabajadores rechazarían el cierre de la empresa, pero de ninguna manera que procederían a intentar enfrentar la ocupación: «el día lunes 21, ante la medida del cierre, no se descartaría una inminente movilización del personal de Astillero (...) hacia el Ministerio de Defensa»

Sólo tras esta consideración, y como una opción poco probable, sugería la posibilidad de que «se intente tomar la planta». Este informe es clarificador porque inicia adelantando que:

«a partir del 21 del Cte. (septiembre) a las 0300 horas, se produciría el cierre del Astillero Río Santiago. Destacándose además que Prefectura Naval Argentina tiene órdenes estrictas que a partir del día y hora señalados no permitan el ingreso de los trabajadores a la planta (...) Es de consignar que (...) la situación descripta (es) hasta el momento desconocida tanto para el sector trabajador como para la dirigencia gremial de ATE Ensenada»

\footnotetext{
${ }^{20}$ Ghigliani, op. Cit.

21 Felipe Venero, "Una mirada a la conflictividad laboral en 1977 a partir del Archivo de Inteligencia de la DIPPBA". IX Seminario Internacional Políticas de la Memoria 40 años del golpe cívico militar: reflexiones desde el presente, 2016, Página 6
} 
El agente explicaba también el plan de la patronal de cierre por 45 días y de reducción de personal a 100 o 150 trabajadores. Replicó aquello que sería difundido al otro día en el comunicado oficial de AFNE y que ya analizamos más arriba. Además, incorporaba el dato de que no solo los trabajadores todavía no habían tomado conocimiento de la decisión de la patronal, sino tampoco el sindicato. Esto es un elemento que luego volveremos a retomar cuando pensemos el propio accionar de los trabajadores. Se adjunta el comunicado de AFNE que dimos cuenta al inicio. El informante concluye comunicando que «se mantendrá un discreto servicio de vigilancia». Este informe corrobora la presunción de que la decisión de la empresa de cerrar sus puertas fue elaborada con anterioridad al propio 18/9 y que recién con la fábrica ocupada se hizo pública tal decisión.

En cuanto a Laboral, también existe un informe del 18 de horas de la mañana. No da cuenta de ninguno de los datos referidos al inmediato de la empresa. Significa que el informante del primer agente era más cercano a la gerencia, mientras que la lectura de este segundo agente sugiere una mirada desde afuera.

Ambos agentes produjeron un informe cerca de las $22 \mathrm{hs}$ del viernes 18 de septiembre. La razón es que desde las $18 \mathrm{hs}$ comenzaron a concentrarse los trabajadores del ARS en 7 y 50 . Los trabajadores, a instancias del Cuerpo de Delegados, convocaron a un acto en homenaje a la Industria Naval para ese día ${ }^{22}$ al que se sumaron activistas estudiantiles, de otros gremios, de fuerzas políticas. Los informes de inteligencia remiten a lo sucedido en el acto. Los dos informes dan cuenta de lo mismo y con pocas variaciones. La concentración comenzó alrededor de las 18:30. Cerca de las 20:50 tomó la palabra el Delegado General del ARS informando sobre la ocupación por el Albatros de la planta y que al día siguiente a las 9 hs se realizaría una asamblea en la plaza central de Ensenada, Plaza Belgrano. A las 21:30 habló el párroco de la Catedral, misa preparada por el Movimiento de Trabajadores Católicos del ARS. Siendo las 21:45 culminó el acto.

El giro de los acontecimientos tomó desprevenidos a los agentes de inteligencia. La convocatoria a la asamblea del día siguiente -sábado- no se encontraba en ninguna de las proyecciones que realizaron los agentes, los cuales esperaban recién acciones el día lunes.

Nuevamente es Gremial quien mejor profundiza el informe del espionaje. En un parte de tres fojas donde se detalla la asamblea convocada a las 9 hs y que inicia a las $10 \mathrm{hs}$ y cada uno de los sucesos subsiguientes - la instalación de carpas en la entrada a la fábrica, el armado de la olla popular, un incidente con camiones que intentaban retirar material de la planta-. Además de la concurrencia de 500 personas, frente a los 250 que aduce el agente de Laboral, en el informe de Gremial puede verse la caracterización o el análisis de la situación que hacían: «no se descarta que el día lunes se realice una asamblea, alrededor de las 07:30hs, con el fin de decidir trasladarse hasta el Ministerio de Defensa» (Mesa B, Factor Gremial, Carpeta 39, Legajo 43, Tomo IV)

La apreciación final del informe choca incluso con los datos que recaba el agente, ya que recoge una de las resoluciones de la asamblea de la mañana donde se define «convocar a todos los trabajadores con el fin de que junto a sus familias se presenten el día lunes (21) a las

${ }^{22}$ El día de la Industria Naval es el 12 de septiembre, pero por problemas técnicos debieron posponer el acto una semana. 
06:30hs en el Astillero Río Santiago con el objeto de preservar y defender las fuentes de trabajo». Es decir, ingresar.

El tercer agente, de Laboral Otras Medidas de Acción Directa, es quizás el único que ofrece un análisis de mayor preocupación. Con informes cortos, ratifica a su superior que «se halla personal del BIM3 y el Grupo Albatros a los fines de preservar las instalaciones ante un posible intento de ocupación del Astillero por parte de los trabajadores». De conjunto, el legajo específico da cuenta de cómo el aparato de seguridad bonaerense no consideraba de ninguna manera que los trabajadores efectivamente fueran a ingresar bajo el riesgo de ser reprimidos por las fuerzas apostadas en la planta. A excepción de estos informes, y uno referido al «Movimiento de Trabajadores Católicos de Astilleros Río Santiago», el resto de los documentos que se encuentran en el legajo son recortes periodísticos y extractos de algunas radios de la región.

Como un actor sin capacidad de intervención en la realidad, los siguientes informes son del lunes 21 y cercanos al mediodía. Tanto Gremial como Laboral relatan los sucesos del domingo 20: asamblea alrededor de las 15:30hs, misa junto al Monseñor Galán y el párroco local y la decisión de ingresar a las 06:00hs del día siguiente. En los dos informes se explica que existió una reunión en horas de la madrugada entre el gobernador Duhalde y el intendente de Ensenada Adalberto Del Negro y que a las 04:30hs los oficiales del grupo Albatros abandonaron las instalaciones del ARS. Esta vez es Laboral el que posee un informe más detallado, al comunicar a su superior que: «alrededor de las 09:00hs se realizó una asamblea de una duración de aproximadamente 20 minutos (...) se decidió efectuar un denominado 'Ensenadazo' sin fecha concreta aún contado con la participación de gremios, partidos políticos y la ciudadanía en general» (Mesa B, Factor Gremial, Carpeta 39, Legajo 43, Tomo IV)

Con la información aquí analizada trabajaba el gobierno -seguramente complementado con otros servicios de espionaje, como por ejemplo la propia Prefectura, de los cuales no se poseen archivos desclasificados-. Dado que la DIPPBA intercambiaba con diversos servicios en operaciones, las conclusiones de este primer análisis es que el gobierno actuó entendiendo que podía, efectivamente, producir un efecto sorpresa a los trabajadores y así proceder a la reducción del 90\% del personal del ARS y transformarlo en un taller de reparaciones dependiente de Zona Franca, tal y como se venía proponiendo en los medios de comunicación.

\section{Enfrentando el cierre}

¿A qué se debió la imposibilidad de los servicios de inteligencia para no poder prever el rumbo definitivo de los acontecimientos? Algunas consideraciones expresadas por los obreros protagonistas arrojan luces sobre este vacío. En su libro sobre la lucha de los trabajadores del Astillero, José Montes relata que «la ocupación militar de la fábrica generó una reacción opuesta a la esperada por el gobierno. Los milicos encontraron una cerrada unidad» y que «nuestra firme decisión de entrar al Astillero a cualquier costo (...) fortalecida por el apoyo y la solidaridad de los trabajadores y estudiantes de la zona, es lo que obligó a que el lunes los 
Albatros abandonaran la fábrica» ${ }^{23}$. Esta evocación de Montes posee un error de temporalidad. Confunde la ocupación de la fábrica y el acto por el Día de la Industria Naval -18 de septiembre- con la fecha en que realmente se conmemora dicho día -12 de septiembre-. Este leve error $^{24}$ permite pensar en aquello que considera Portelli ${ }^{25}$ sobre los testimonios y la memoria no como un reservorio de datos precisos sino como apreciaciones verosímiles.

La conclusión de Montes de que «la ocupación militar de la fábrica generó una reacción opuesta a la esperada por el gobierno» se ve ratificada por la crónica que hace Raúl Corzo ${ }^{26}$. Allí relata cómo, al finalizar el acto del 18, los delegados se reunieron en el local de ATE Provincia y discutieron el acampe frente a la empresa que llamarían a votar el día siguiente. Agrega, exagerando, que «otra vez los estrategas del vaciamiento se equivocaron. Los 1370 compañeros que no nos habíamos anotado en el retiro voluntario reaccionamos rápido, acampamos en la puerta y lanzamos una fuerte campaña de agitación por La Plata, Berisso y Ensenada» ${ }^{27}$. En la noche del 19 de septiembre, los delegados, junto a la dirección de ATE Provincia y Nación, se reunieron en la cantina de "Carlitos" y discutieron sobre si ingresar o no. La posición del gremio provincial y nacional era aguardar al desgaste general; los delegados defendieron ingresar y, si se reprimía, que así fuera.

Lo escrito por Corzo se completa con lo que plantea Carlos Raymundo acerca del debate que rondaba la asamblea de las 10 de la mañana del sábado 19 en plaza Belgrano:

«habíamos llegado a la conclusión de que el que negocia con Menem pierde. "ramal que para, ramal que cierra". Se pierde. Nosotros sabíamos que íbamos a la guerra. Ya se había discutido hasta el cansancio. Sobre la experiencia de lo que había pasado en Somisa en mayo, el Cuerpo de Delegados del ARS, sobre todo la Mesa, llega a la conclusión del que negocia, pierde.

Y ahí se decide el sitio del ARS por afuera» (Entrevista a Raymundo, 27/5/2017)28

Raymundo explica otro de los límites que ya observamos en las apreciaciones de los servicios de inteligencia. Al momento de realizarse el acto del día de la Industria naval, la Mesa de Delegados ya estaba enterada de la llegada del Albatros: «Viene una piba, la Negra Neca,(...). Ella laburaba en Computación en la parte de liquidación de sueldos. Viene y nos dice 'Che, vino el Coco Álvarez, con la pistola en la cintura y un capitán de infantería y nos dijo que nosotros tenemos que laburar todo el fin de semana haciendo la liquidación solos'. Habían armado todo. Entonces, transformamos el acto en una denuncia política» (Entrevista a Raymundo, 27/5/2017)

\footnotetext{
${ }_{23}$ Montes era delegado de Sección y militante de la Lista Marrón. Véase José Montés “Astillero Río Santiago: su bistoria y su lucha relatada por sus trabajadores”. Buenos Aires: La verdad obrera, 1999. Página 88

24 Importante en cuanto hay investigaciones que se aferraron a estas fechas sin consultar otros testimonios.

25 Alessandro Portelli, "Historia y memoria: la muerte de Luigi Trastulli". Historia y fuente oral, 5-32, 1989.

${ }^{26}$ Corzo era Delegado de Sección en ese entonces y militante de la Lista Naranja. Raúl Corzo, "Un sentimiento llamado Astillero". Buenos Aires: Dunken, 2010. Página 463.

${ }^{27}$ Corzo, op. Cit. Página 465. Ninguno de los informes de la DIPPBA que aquí se analizan informan más de 500 participantes de la asamblea del sábado 19 de septiembre.

${ }_{28}$ Raymundo era Delegado de la sección Buques Militares, miembro de la Mesa de Delegados electa en agosto de ese año y militante de la Lista Violeta.
} 
Otro de los trabajadores se posiciona desde la misma tónica:

«Decidimos que íbamos a entrar. Ese día (20/9) yo había organizado con la iglesia para que den una misa en apoyo, en asamblea discutimos ingresar. Justo había venido el obispo al campamento. Se reunió con las mujeres. Iba a correr sangre, nosotros el lunes entrábamos. Y entonces ayudaron, explicaron eso y los Albatros se fueron. Nos gritaban (los Albatros) 'ya van a ver cuando volvamos' (...) de impotencia. Pero íbamos a entrar, estábamos dispuestos a todo. Nuestras familias iban a entrar, los curas, todos. Fue, por así decir, una casualidad» (Entrevista a Cacho, 22/6/2017) ${ }^{29}$

Agrega como virtud de la experiencia de debate en aquellos momentos el hecho de que «las diferencias se discutían en las asambleas, en especial las de sector. Había muy buenos oradores y las asambleas siempre eran por la positiva»

Todos los testimonios, recientes o lejanos -como el de Montes- parten de dos consideraciones. Por un lado, la decisión de ingresar a la fábrica para defender las fuentes de trabajo porque «el que negocia con Menem pierde», en palabras de Raymundo. Por el otro, la unidad de acción que significaba la posibilidad de intervenir de manera coordinada el conjunto de los trabajadores y sus instancias de organización. Esta unidad de acción se expresó en la propia conformación de la Mesa de Delegados electa un mes antes de la ocupación por parte del Albatros. Eran parte de la misma trabajadores de la Lista Bordó, vinculada al Partido Comunista Revolucionario (PCR); la Blanca vinculada al PJ; la Violeta del Partido Obrero (PO); otro que, sin decirlo, estaba cercano a la Azul y Blanca de la vieja conducción peronista de la época del golpe; y un sexto cercano al radicalismo. Incluso Corzo, vinculado al Movimiento de los Trabajadores Socialistas (MST) y opositor en ese entonces a dicha Mesa, afirma que «como equipo de conducción, tuvo una actuación en defensa de la fuente de trabajo, a mi entender, inobjetable (...) hubiera hecho similares acciones a las (que) ellos tomaron $»^{30}$. Ninguno de estos elementos es informado por los agentes de inteligencia.

\section{¿Una organización obrera particular?}

La organización de los trabajadores del Astillero partía de la asamblea por sección y la asamblea general; cada sección elegía sus delegados de sección que constituían el Cuerpo de Delegados. De allí se votaba la Mesa de Delegados o Comisión Interna. Además, el Astillero formaba parte de (ATE), cuya seccional local incluía dependencias estatales no fabriles de Ensenada. La Mesa de Delegados durante este suceso específico de la ocupación del Albatros era opositora a la conducción de la seccional ATE Ensenada fundamentalmente en la forma de la organización interna ${ }^{31}$.

${ }^{29}$ Cacho había miembro de la Comisión Administrativa Seccional y era Delegado de Sección en aquel entonces, militante de la Lista Blanca. Prefirió no ser entrevistado con su nombre real.

${ }^{30}$ Corzo, op. Cit. Página 455.

${ }^{31} \mathrm{Al}$ respecto pueden verse los trabajos de María de las Nieves Piovani "Estrategias de lucha de ATE y el Astillero Río Santiago frente a la embestida privatista y reformadora del primer gobierno de Menem”. La Plata: UNLP, 2002; y Cecilia Pérez Pradal, "Contra el naufragio. Un estudio sobre los conflictos laborales en el caso del Astilleros Río Santiago 1989-1999”. La Plata: UNLP, 2002. 
Juliana Frassa ${ }^{32}$ analiza este problema de la dificultad del gobierno para proceder al cierre o privatización del Astillero y concluye que uno de los factores importantes a tener en consideración es la ausencia de un posible comprador del Astillero, lo cual imposibilitaba la privatización de la empresa. Incorpora la necesidad de los gobiernos nacional y provincial de retirar de la esfera pública el conflicto casi permanente con el ARS que realizó decenas de movilizaciones a la Capital Federal entre 1989 y 1993. Estos factores, junto a la propia lucha de los trabajadores, llevaron a buscar una salida negociada entre todos los actores: la Zona Franca con todo el personal adentro. Sin embargo, fue recién con el fracaso del intento de cierre del 18 de septiembre de 1992, retirada de las tropas de ocupación mediante, que se definió esta salida, y con un año de posterioridad. Grandes diarios como Crónica titularon «Se impusieron los obreros» ${ }^{33}$.

A partir de los informes de los agentes de inteligencia de la DIPPBA, en contraste con los testimonios de algunos de los trabajadores, es dable afirmar, introductoriamente, que el servicio de espionaje tenía como límite los procesos de organización y de discusión precisos; incluso instancias cerradas de "conspiración" como aquella reunión en ATE Provincia al terminar el acto del 18 de septiembre. Ninguno de los informes da cuenta de la misma. Se limitan a señalar la desconcentración a las 21:30hs. Evidentemente, entre los delegados y el activo de la fábrica no había ningún informante de la DIPPBA.

\section{Unas primeras conclusiones: ¿por qué no se cerró la fábrica?}

Carlos Raymundo señala en determinado momento de la entrevista que «si nos sacaban, iba a ser con los pies para delante». Esta reflexión permite aproximarse a la subjetividad de parte de ese colectivo obrero que se enfrentó a la ocupación militar de su lugar de trabajo. El gobierno procedió con el desconocimiento de los vacíos en los informes y, quizás, con la convicción de que de ninguna manera un sector de los trabajadores se opondría a su política hasta las últimas consecuencias.

Los agentes de inteligencia no fueron capaces de insertarse en la propia organización de los trabajadores del Astillero. Por más que hubiera algún informe que pudiera llegar sobre las resoluciones de las asambleas, en ningún momento fueron capaces de registrar las discusiones y definiciones de espacios más acotados como los cuerpos de delegados, las reuniones de la Mesa y, ni hablar, instancias semi-clandestinas como la reunión posterior al acto del 18 de septiembre. Gracias a esto -hemos podido comprobar- los trabajadores actuaron por fuera de toda previsión tanto de los servicios de inteligencia como del propio gobierno

En su introducción a Lo que todo revolucionario debe saber sobre la represión, Serge reflexiona la frase que da inicio al presente artículo; según él, sin importar la ciencia y los crímenes que realicen, todas las policías son impotentes ante la marea revolucionaria en su ascenso. Sus conclusiones parten del análisis que hace de los archivos de inteligencia de la Ojrana, el servicio

\footnotetext{
32 Juliana Frassa, "Evitando el naufragio de la privatización: Estrategias laborales y empresariales en la industria naval frente a la transformación del contexto socio-económico. El caso del Astillero Río Santiago en los años 90”. Buenos Aires: Tesis Maestría, 2009.

${ }^{33}$ Crónica, 22/9/1992
} 
de inteligencia del zarismo, abierto cuando se produjo la Revolución de Octubre de 1917 en Rusia.

La conclusión a la que arriba Serge obliga a abrir preguntas sobre, justamente, todas aquellas instancias de discusión y organización a las cuales el Estado era incapaz de ingresar a través de sus mecanismos de espionaje. Los protagonistas de aquellos sucesos poseen la llave para incursionar sobre esos problemas. La derrota del intento de cierre del Astillero es una muestra de ello. El destino ulterior de la fábrica, es otra historia.

\section{Bibliografía}

Fuentes primarias

COMISIÓN PROVINCIAL POR LA MEMORIA. Mesa B, Factor Gremial, Carpeta 39, Legajo 43, Tomo IV. 315 folios.

CORZO, Raúl. Un sentimiento llamado Astillero. Buenos Aires: Dunken. 2010

MONTES, José. Astillero Río Santiago: su bistoria y su lucha relatada por sus trabajadores. Buenos Aires: La verdad obrera. 1999

Entrevistas

Carlos Raymundo. Entrevista realizada el 27/5/2017 en su casa.

Cacho. Entrevista realizada el 22/6/2017 en el lugar de trabajo.

Bibliografía

AIZICZON, Fernando. "Trayectorias militantes, izquierda y política sindical: la intervención del MAS en Sierra Grande a través de las vivencias de un obrero minero, Patagonia Argentina (19881991)". En: Izquierdas $\mathrm{N}^{\circ}$ 31. 46-70 (diciembre, 2016). Disponible en: https://scielo.conicyt.cl/scielo.php?script=sci arttext\&pid=S0718-50492016000600046

AZPIAZU, Daniel. Las privatizadas: ayer hoy y mañana. Buenos Aires: Capital Intelectual. 2005.

BRESSER PEREIRA, Luiz Carlos. (1998). "La reforma del Estado en los años noventa. lógica y mecanismos de control”. Desarrollo Económico vol $38 N^{\circ} 150$ (julio-septiembre, 1998), 517-550. Disponible en: http://abcdonline.com.ar/tea/info/TEA0255.pdf

BONNET, Alberto. La hegemonía menemista: el neoconservadurismo en Argentina, 1989-2001. Buenos Aires: Prometeo. 2008

BORON, Atilio y THWAITES REY, Mabel. "La expropiación neoliberal. El experimento privatista en la Argentina”. En PETRAS, James y VELTMEYER, Henry, Las privatizaciones y la desnacionalización de América Latina. Buenos Aires: Prometeo. 2004. 113-182.

CASTILLO, Fernando, BERGESIO, Liliana y GOLOVANEVSJY, Laura. "Trabajadoras de Altos Hornos Zapla: de la casa a la fábrica, del estado a la privatización". II Jornadas Nacionales sobre Estudios Regionales y Mercados de Trabajo. (4 y 5 julio, 2012). Disponible en: http://www.simel.edu.ar/wpcontent/uploads/2016/05/02-M5-castilloBergesioGolovanevsky-Ponencia.pdf

ESPONDA, María Alejandra. "Debates, formas de organización y disputas de poder en Propulsora Siderúrgica durante la reestructuración productiva de los 90". III Jornadas de Economía Politica. (9, 10, 11 noviembre, 2009). Disponible en: $\quad$ http://www.ungs.edu.ar/ms idh/wpcontent/uploads/2009/11/Maria_Alejandra_Esponda_Debates formas de organizacion_y disputas. pdf 
"La reestructuracion productiva de los 90 en Propulsora Siderúrgica: debates, formas de organización y disputas de poder". En BASUALDO, Victoria. La clase trabajadora argentina en el siglo XX: experiencias de lucha y organización. 2011. Buenos Aires: Cara o Ceca. 325361

FRASSA, Juliana. (2006). "Políticas públicas de desregulación y sus consecuencias sobre la producción y el empleo. El caso de la política naviera y la industria naval argentina". Informe IEFE, 3951.

Evitando el naufragio de la privatización: Estrategias laborales y empresariales en la industria naval frente a la transformación del contexto socio-económico. El caso del Astillero Río Santiago en los años 90. Buenos Aires: Tesis Maestría. 2009

Estrategias de resistencia laboral frente a la política privatizadora neoliberal. Reflexiones a partir del estudio de una empresa industrial. Theomai. $\mathrm{N}^{\circ} 22$ (septiembre, 2010). Disponible en: http://revista-theomai.unq.edu.ar/numero\%2022/Art Frassa.pdf

FRASSA, Juliana y RUSSO, Cintia (2012). "Trayectoria reciente y perspectivas futuras de la industria naval pesada argentina: Los astilleros estatales". Revista de estudios regionales y mercado de trabajo. $\mathrm{N}^{\circ}$ 8 (2012). 77-98. Disponible en: http://www.memoria.fahce.unlp.edu.ar/art revistas/pr.5587/pr.5587.pdf

GHIGLIANI, Pablo. “Archivos policiales e historia social del trabajo (1957-1977)”. VII Jornadas de Sociologia UNLP. La Plata (diciembre 2012). Disponible en: http://sedici.unlp.edu.ar/handle/10915/30743

JAITTE, Pablo. La lucha de los petroleros contra la privatización de YPF: Un intento de síntesis. VI Jornadas de sociología UNLP. La Plata (2010). Disponible en: https://www.aacademica.org/000027/198.pdf

KAHAN, Emmanuel. ¿Qué represión, qué memoria? El "archivo de la represión” de la DIPBA: problemas y perspectivas. Question. Vol 1. $\mathrm{N}^{\circ} 16$ (2007). Disponible en: http://perio.unlp.edu.ar/ojs/index.php/question/article/view/459

LANTIERI, Magdalena y NAZAR, Mariana. (2014). A 10 años de la apertura del Archivo de la DIPPBA. Aletheia. Vol 5 N $\quad$ N $\quad$ Disponible http://www.aletheia.fahce.unlp.edu.ar/numeros/numero-9/conferencias/a-10-anos-de-la-apertura-delarchivo-de-la-dippba.-primer-panel-presentacion-de-los-instrumentos-de-descripcion-del-fondodocumental-de-la-ex-dippba

MARENGO, María Eugenia. Los mecanismo del control social: el caso de la ex DIPBA. Derecho y Ciencias Sociales $\mathrm{N}^{\circ} 4$ (abril, 2011), 147-162. Disponible en: http://sedici.unlp.edu.ar/handle/10915/15272

MAURO, Lucía Mercedes.La industria naval como un sector estratégico: análisis del rol Estado. VI Jornadas de Jóvenes Investigadores. UNMdP (10, 11 y 12 noviembre 2011). Disponible en: http://nulan.mdp.edu.ar/2544/1/mauro.2011.pdf

NOVARO, Marcos. Argentina en el fin de siglo. Democracia, mercado y nación (1983-2001). Buenos Aires: Paidos. 2009.

OSZLAK, Oscar. El mito del Estado mínimo: una década de reforma estatal en Argentina. Desarrollo Económico vol $42 \quad N^{\circ} 168$ (enero-marzo 2003). Disponible en: http://www.oscaroszlak.org.ar/images/articulosespanol $/ \mathrm{El} \% 20$ mito $\% 20 \mathrm{del} \% 20$ Estado $\% 20$ minimo $\% 20 \mathrm{una} \% 20 \mathrm{dec} \% 20 \mathrm{de} \% 20 \mathrm{ref} \% 20 \mathrm{est} \% 20 \mathrm{en} \% 20 \mathrm{Ar}$ g.pdf

PALERMO, Hernán. Cadenas de oro negro en el esplendor y ocaso de YPF. Buenos Aires: Grupo Antropología del Trabajo. 2012

PÉREZ PRADAL, Cecilia. Contra el naufragio. Un estudio sobre los conflictos laborales en el caso del Astilleros Río Santiago 1989-1999. La Plata: UNLP. Tesis de grado. 2002 
PIOVANI, María de las Nieves. Estrategias de lucha de ATE y el Astillero Río Santiago frente a la embestida privatista y reformadora del primer gobierno de Menem. La Plata: UNLP. 2002. Disponible en: http://www.memoria.fahce.unlp.edu.ar/tesis/te.632/te.632.pdf

PIVA, Adrián. El desacople entre los ciclos del conflicto obrero y la acción de las cúpulas sindicales en Argentina (1989-2001). Estudios del Trabajo Nº31 (enero-junio 2006), 23-52. Disponible en: https://www.academia.edu/30506726/ El desacople entre los ciclos del conflicto obrero y la ac ci $\%$ C3\%B3n de las c\%C3\%BApulas sindicales en Argentina 19892001 en Revista Estudios del Trabajo ASET Buenos Aires primer semestre de 2006 pp. 23-52 Acumulación y begemonía en la Argentina menemista. Buenos Aires: Biblos. 2012.

PORTELLI, Alessandro. Historia y memoria: la muerte de Luigi Trastulli. Historia y fuente oral. $\mathrm{N}^{\circ}$ 1 (1989). 5-32. Disponible en: http://etnohistoriaenah.blogspot.com/2010/10/portelli-historia-ymemoria-la-muerte.html

POZZI, Pablo y SCHNEIDER, Alejandro. Combatiendo al capital: crisis y recomposición de la clase obrera Argentina 1983-1993. Buenos Aires: El Bloque. 1994.

RUSSO, Cintia. Trayectorias de una empresa del estado: Astilleros Río Santiago. XXI Jornadas de Historia Económica. UNTREF $(23$ al 26 septembre, 2008). Disponible en: http://xxijhe.fahce.unlp.edu.ar/programa/descargables/russo

Astillero navales del Estado. XXII Jornadas de Historia Económica - Río Cuarto. 2010.

SERGE, Víctor. Lo que todo revolucionario debe saber sobre la represión. Buenos Aires: Madreselva. 2010

SOLARI PAZ, Ana Cecilia. (2016). Para publicar en actas. 82. Estado, politica y sociedad en una Argentina en crisis (1955-1989). 2016

SOUL, María Julia. Transformaciones de las estrategias sindicales en contextos de Reconversión Productiva. Un estudio de caso en la Industria Siderúrgica. Buenos Aires: Campus CLACSO. Buenos Aires. 2007. 1-30

TORRES, Fernanda. La privatización de YPF en Comodoro Rivadavia. Algunas características y consecuencias sociales y laborales. Trabajo y Sociedad. Nº18 (enero-junio, 2012), 279-295. Santiago del Estero. Disponible en: http://www.scielo.org.ar/scielo.php?script=sci arttext\&pid=S1514$\underline{68712012000100018}$

VENERO, Felipe. Una mirada a la conflictividad laboral en 1977 a partir del Archivo de Inteligencia de la Policía de la Provincia de Buenos Aires. IX Seminario Políticas de la Memoria. CCM Haroldo Conti. Buenos Aires. 2016. 